Seta, J. J., Seta, C. E., \& McElroy, T. (2003). Attributional biases in the service of stereotype maintenance: A schema-maintenance through compensation analysis. Personality and Social Psychology Bulletin, 29: $151-163$. Published by SAGE (ISSN: 0146-1672).

\title{
Attributional Biases in the Service of Stereotype Maintenance: A Schema-Maintenance Through Compensation Analysis
}

\author{
John J. Seta, Catherine E. Seta, and Todd McElroy
}

\begin{abstract}
Six experiments were conducted to test assumptions of a schemamaintenance through compensation analysis. The results of these experiments indicated that perceivers can compensate for the inconsistent action of one individual (the target) by altering their attribution concerning the action of a fellow group member. When the target performed an inconsistent behavior, perceivers compensated by making especially extreme stereotypically consistent attributions concerning a fellow group member's subsequent action. In addition, in Experiment 5, perceivers compensated via a fellow group member for a target's inconsistent action while maintaining their general view of group members. Experiments also provided tests of the capability and motivation assumptions of the schema-maintenance through compensation analyses.
\end{abstract}


A vast body of research on attributional processes has generally assumed that causal analysis of a person's behavior is motivated by the person's need to predict the future (see Fiske \& Taylor, 1991, for a review; see also Heider, 1958; Jones \& Davis, 1965; Kelley, 1973). Understanding the dispositional or situational forces that are associated with behavior enables people to feel that they can control or at least predict events. Thus, attributions are assumed to serve a functional role, contributing to a person's sense of self-efficacy. In addition, attributions serve a role in interpersonal relations in influencing, for example, the course of social interaction including close relationships and intergroup behavior. The use of stereotypes is also functional in this context in making the social world more predictable (e.g., Allport, 1954; Fiske\& Taylor, 1991). However, functional utility comes with a social cost; stereotypes often are maintained in the face of disconfirming evidence that can lead to the perpetuation of prejudice and bias.

Research has shown that the type of attribution a perceiver makes can influence whether an aberrant behavior has an impact on a perceiver's representation of the stereotyped group (e.g., Crocker, Hannah, \& Weber, 1983; Hewstone, 1989). For example, Crocker et al. (1983) found that the type of attribution that was made about an inconsistent action influenced the impact of the inconsistent action on memory for the action and representations of the behavior. The aberrant act had a stronger influence on changing representations of social groups when it was described as being internally, rather than externally, motivated. Further research has demonstrated that stereotypes are most likely to change when counterstereotypic behaviors are performed by group members thought to be typical members of outgroups and when these behaviors are attributed to stable, internal causes (e.g., Wilder, Simon, \& Faith, 1996).

There are several perspectives that implicate attributions in the process of stereotype maintenance/change (e.g., Crocker et al., 1983; Hewstone, 1989; Johnston, Bristow,\&Love, 2000; J. J. Seta\&Seta, 1993; Wilder et al., 1996; Yzerbyt, Rogier, \& Fiske, 1998). Thus, exploring the factors that influence the outcomes of attributional search processes is an important area of investigation. In our research, we focused on the role of category-based expectancies on individuals' attributions and perceptions of a behavior that followed exposure to stereotypically inconsistent acts. Specifically, we were interested in determining if the nature of perceivers' attributions was 
influenced by their propensity to compensate for the stereotypically inconsistent behaviors of a group member. The present research follows from our previous work on compensatory motives in social judgment. First, we describe this compensatory motivation analysis followed by a description of the current research.

\section{SCHEMA-MAINTENANCE THROUGH COMPENSATION}

A schema includes goals, expectations, self-concepts, stereotypes, moods, and other such organizing concepts and motives. Compensation is not restricted to one type of schema or one type of motivation. For example, people can compensate in an attempt to maintain a goal, to maintain control, or to maintain a particular view of themselves or others (e.g., J. J. Seta, Seta,\&Erber, 1993; J. J. Seta \& Seta, 1993). When individuals attempt to maintain a schema, they are effectively trying to prevent a schema from changing. Thus, this model has particular relevance to situations in which individuals attempt to preserve their stereotype in the face of disconfirming evidence.1 In this article, we are concerned with stereotype maintenance. According to our view, exposure to counterstereotypic behaviors can result in the generation of hyperconsistent expectations that the group member will conform to the stereotype in the future by performing a behavior that is hyperconsistent with the stereotype (J. J. Seta\&Seta, 1993). For example, observations of a member of a social group, typically thought to be lacking in intelligence, performing an intellectual task may lead individuals to expect that this group member would be likely to perform in a manner especially lacking in intellectual acuity in the future. This expectation may effectively help maintain the negative group stereotype. Similarly, individuals may attempt to maintain positive stereotypes by generating compensatory expectations, such as when observations of a minister behaving in an immoral manner lead individuals to expect the minister to behave in an ultrarighteous manner at a future date. This expectation may or may not effectively counter the influences of observed inconsistencies. Regardless of whether the generation of compensatory expectations effectively maintains the stereotype, we should observe the influences of this motive on subsequent social judgments under certain conditions.

According to our schema-maintenance through compensation approach, compensation can occur when an 
event or experience is subjectively inconsistent with a stereotype or schema (J. J. Seta \& Seta, 1993; J. J. Seta, Seta, \& Erber, 1993). In this situation, compensation will occur when a person has an implicit or explicit motivation to maintain the stereotype or schema and the person has the capability of generating information (or behavior) that could potentially offset the inconsistency. In support of this assertion, research has demonstrated that cognitive capacity is necessary for stereotype maintenance in the face of receiving inconsistent information about a target (e.g., Moreno \& Bodenhausen, 1999; Yzerbyt, Coull, \& Rocher, 1999). Individuals are especially willing to expend cognitive effort in generating compensatory information when, for example, important or valued schema are threatened by inconsistent information. Therefore, within limits, one's capability of generating information may vary as a function of schema importance.

Perceivers' motivation to compensate should be influenced by factors such as the schema's subjective importance (C. E. Seta, Seta, \& Goodman, 1998), schema strength, and the consequences of maintaining this schema. There are also several factors that constrain a perceiver's capability of accessing or generating potentially compensating information. These include factors such as the frequency and intensity of observed inconsistency. As evidence mounts that disconfirms expectations, it becomes increasingly difficult to disregard the data (e.g., J. J. Seta \& Seta, 1982, 1992; Wilson, Lisle, Kraft, \& Wetzel, 1989). At this point, expectations may not be completely guided by schematic knowledge, and predictions about the deviant target's behavior may be guided by bottom-up observations of the target's behavior. In this case, the perceiver is basing his or her expectations on the observed behavior of the deviant target, and perceivers will not expect compensation from the deviant actor.

According to our view, even though the perceiver is unable to compensate for a target's inconsistent actions, it is possible that the perceiver will expect compensation from a different group member. A fellow group member and the target of the inconsistent action are related in a perceiver's knowledge structure (e.g., Fiske \& Neuberg, 1990; Fiske \& Taylor, 1991). Therefore, perceivers may attempt to maintain their stereotype by expecting the future action of a nondeviant group member to compensate for the inconsistent actions of a deviant member. 
There are several reasons why a perceiver can expect compensation from a fellow group member under conditions in which he or she does not expect compensation from a deviant target. First, the assumed variability among members of a group is often greater than the assumed variability in a single individual's behavior. For example, we generally are not surprised when grades in our class range from As to Fs, but we are surprised when the grades of a single student range from As to Fs. People often expect more behavioral deviance among a group of people than within a single individual (e.g., Hamilton \& Sherman, 1996). This effect also can occur because the implications of the inconsistent actions of the deviant are more directly tied to the personality of the deviant target than to the personality of a fellow group member. For both reasons, it should take an objectively less severe inconsistent action to constrain a perceiver's capability of generating compensatory expectations about the target of the inconsistent actions than a fellow group member's future actions. Likewise, it should take an objectively milder inconsistent action to generate compensatory expectations for the target than a fellow group member. It follows, then, that after a very inconsistent action on the part of the target, we may observe compensation for a fellow group member but not for the target.2

These predictions were supported in a series of experiments (C. E. Seta et al., 1998; J. J. Seta \& Seta, 1993). In one experiment, for example, we found that after a target performed a very mild inconsistent action, participants generated compensatory expectations about the future action of the target; however, following a highly inconsistent action, participants generated compensatory expectations for the fellow group member but not for the acting target. Just as perceivers demonstrate compensatory motivation by elevating their future expectations of a group member's actions, the type of attribution they make about a group member's actual behavior also may reflect this motive. In particular, individuals may be biased toward making more extreme internal attributions for a group member cohort's stereotypically consistent behavior in an effort to maintain their existing group schema. Thus, an influence of compensatory motives on patterns of attributions would be demonstrated by finding more extreme dispositional attributions for stereotypically consistent behaviors in the presence versus the absence of inconsistency. The present research explored this possibility. 


\section{OVERVIEW: EXPERIMENTS 1 AND 2}

In these studies, we explored the viability and generalizability of the schema-maintenance through compensation analysis with respect to participants' interpretations and attributions of potentially compensatory behaviors. In all studies, participants were presented with the aberrant behavior of a member of a stereotyped group, and positive group stereotypes were used that are typically associated with either women (nurses) or men (ministers). Experimental condition participants were exposed to stereotypically inconsistent information about a member of one of these groups and were asked to make judgments about the causes of a stereotypically consistent behavior that was described as emanating from the aberrant group member (same target conditions) or a fellow member of the target's social group (fellow group member conditions). In addition, control conditions were employed in which participants were not exposed to stereotypically inconsistent behaviors and were asked simply to make judgments about the causes of the stereotypically consistent behaviors. These controls allowed for an estimate of baseline attributions used for reference points in assessing the extent to which exposure to inconsistency changed patterns of attributions. An influence of compensatory motives on patterns of attributions would be demonstrated by finding more extreme dispositional attributions for stereotypically consistent behaviors in experimental versus control conditions. We expected to see more extreme attributions about the causes of fellow group members' stereotypic behavior, which are consistent with the disposition of the actor, after being exposed to the inconsistent behaviors of a target group member. Participants would be indicating stronger stereotypically consistent dispositional causes for the behavior than baseline estimates. The primary purpose of these studies was to demonstrate the generalizability of the hypothesized effects across materials and stereotypes. Further studies, reported in this article, were designed to rule out potential alternative explanations, such as contrast effects. These findings would dovetail with prior research (e.g., C. E. Seta et al., 1998; J. J. Seta \& Seta, 1993) that has found increased expectations for stereotypically consistent behaviors on the part of fellow group members after exposure to highly inconsistent behaviors of an acting group member. 


\section{METHOD: EXPERIMENTS 1 AND 2}

\section{Stimulus Materials}

In all experiments, inconsistent behaviors were chosen from a normative set collected for prior research

(J. J. Seta\&Seta, 1993) and were considered to be highly inconsistent with the stereotyped group. The target compensatory behavior used in Experiments 1 and 2 also was selected from these norms. In addition, a separate sample of participants was given the target compensatory behavior used in these studies. They were asked to assume that either a minister (Experiment 1) or a nurse (Experiments 2-6) had performed a behavior and were asked if this behavior was consistent or inconsistent with the internal character of the person's group. For ministers, they were asked to assume that a minister volunteered, in the presence of other ministers, to help the March of Dimes (a charitable, philanthropic group) over the weekend. Then, they were asked which of two conclusions for this behavior (he did it because he was totally concerned about the March of Dimes or he wanted to make a good impression) was most consistent and which was most inconsistent with the internal character of ministers. Of the 30 participants that were sampled, 27 believed that the conclusion that suggested that the minister helped because he was concerned about the March of Dimes was most consistent with the character of ministers, $X^{2}=19.2, p<.001$, whereas 28 believed that the conclusion that he wanted to make a good impression was most inconsistent with the character of ministers, $X^{2}=22.5, p<.001$. For nurses, participants were asked to assume that a nurse volunteered, in the presence of other nurses, to help the March of Dimes over the weekend. Then they were asked which of two conclusions (she did it because she is a truly generous person or because she simply wanted to impress) was most consistent and which one was most inconsistent with the internal character of nurses. Of the 30 participants, 28 believed that the conclusion that she was truly generous was most consistent, $\mathrm{X}^{2}=22.5, p<.001$, whereas 29 believed that the conclusion that she wanted to impress was most inconsistent, $X^{2}=26.1, p<.001$.

Participants, Design, and Procedure (Experiments 1 and 2)

Experiment 1. Ninety-one women and 21 men participated in Study 1. No participant was included as an overlapping participant in any of the studies reported in this 
article. Participation was rewarded by the receipt of partial credit for the research requirements of introductory psychology classes at the University of North Carolina, Greensboro.

In Experiment 1, participants were assigned randomly to one of four between-subjects conditions defined by the factorial crossing of two levels of actor inconsistency (a high-inconsistency experimental condition and no inconsistency control) with two levels of target identity variable (aberrant group member and a fellow member condition). The study was conducted in small groups where the number per group typically ranged from 8 to 12. Participants were told that, in the present study, they would be asked their impressions of other persons. They were then given written descriptions comprising one of the four conditions used in the study; all conditions were approximately equally represented within each experimental session.

Participants in the high-inconsistency experimental condition read about a minister who had sexually molested a 7-year-old and sold heroin to a teenager. In the same aberrant group member condition, they then read that this minister volunteered in the presence of other ministers to help the March of Dimes in the forthcoming months. They were then asked to consider why this minister had volunteered; they rated their attribution along a 7-point scale anchored by the endpoints 1 (to make a good impression) and 7 (he is totally concerned about the March of Dimes).

Participants in the fellow group member condition read about the inconsistent behaviors of the aberrant minister but were told that another minister, unrelated to the inconsistent actor, volunteered to help the March of Dimes. They were then asked to rate the reason why he volunteered along the 7-point scale described above. Participants in the two control groups were not exposed to inconsistent information about ministers and were simply asked to rate the reasons why a minister would volunteer or why a different minister would volunteer. All participants were asked a probe question serving as a manipulation check in which they were asked to indicate the identity of the rating target. Following this question, participants were thanked and debriefed.3 Experiment 2. Similar procedures were used in Experiment 2. However, this study employed 73 female participants. In the high-inconsistency conditions, participants read a scenario in which they were asked to imagine finding a nursing student described as red-faced and grasping 
a frail elderly man by the collar while slapping him in the face. A broken glass was described as lying close-by on the floor in a puddle of milk. They then read that later that week, a member of the community asked Mary to donate a great deal of time on a Saturday to help a charitable organization. This request was described as made in the presence of other persons.

At this point, our manipulation of the attributional target was made. Participants in a high-inconsistencysame target condition read that Mary was the nursing student that they had just read about in the preceding scenario (i.e., the aberrant nurse), whereas participants in a high-inconsistency-different target condition read that Mary was not the nursing student described in the preceding scenario but was a different nursing student from the one who performed the aberrant actions. Participants were then asked to rate their attributions for why Mary volunteered along a similar 7-point scale used in Experiment 1.

To obtain a baseline estimate of the extent to which volunteering was considered to be a dispositional/situational motive for nursing students, an additional group of participants was asked simply to assume that Mary was a nursing student who was asked to volunteer without exposure to any stereotypically inconsistent information. Thus, the design of Experiment 2 included three between-subject conditions (high-inconsistency-same target; high-inconsistency-different target; no inconsistency control). In addition to the probe question used in Experiment 1, participants in this study were asked whether they were nursing majors. 4

\section{RESULTS AND DISCUSSION: EXPERIMENTS 1 AND 2}

Participants' rating scores in Experiment 1 were entered into a 2 (group member identity) $\times 2$ (inconsistency) between-subjects ANOVA. The analysis revealed a main effect of the target identity variable, $F(1,101)=$ 25.97, $p<.001$, and a marginally significant inconsistency main effect, $F(1,101)=2.94, p<.09$. These effects were qualified by a Target Identity $\times$ Inconsistency interaction, $F(1,101)=39.80, p<.001$. This interaction was interpreted via planned comparisons. As expected, participants who read about the aberrant behaviors of the minister attributed his generosity in volunteering to the dimension that was more inconsistent with the dispositional attribution of ministers-impressing others 
$(M=2.26)$-in contrast to the same target control participants $(M=4.62), F(1,101)=34.06, p<.01$. On the other hand, participants in the fellow group member condition attributed the other minister's generosity to the dimension that was more consistent with the disposition of ministers $(M=5.61)$ in contrast to comparable control participants who were not exposed to the inconsistent actions $(M=4.26), F(1,101)=10.31, p<.01$. Thus, exposure to the previous aberrant behavior of a minister produced stronger dispositional attributions for another group member's stereotypically consistent behavior.5

A conceptually similar pattern of results was obtained in Experiment 2. The results of the one-factor between subjects ANOVA revealed significant differences between conditions, $F(2,58)=39.23, p<.0001$. Planned comparisons among the cell means indicated that participants weighted factors that were inconsistent with the dispositional character of nurses more heavily in their attributions of the nursing student's charity work when she was described previously as performing stereotypically inconsistent behaviors $(M=1.75)$ than did control participants $(M=3.81), F(1,58)=33.59, p<$ .001. In contrast, participants who had read about the aberrant behavior of the nursing student rated the charity work of another nursing student as more consistent with nurses' dispositional character $(M=4.89)$ than did control participants $(M=3.81), F(1,58)=9.23, p<.01$. Thus, Experiment 2 provided a conceptual replication of Experiment 1 in demonstrating a role of compensatory motives on attributions of causality.

\section{INTRODUCTION: EXPERIMENT 3}

The results of Experiments 1 and 2 dovetail with our previous research (e.g., C. E. Seta et al., 1998; J. J. Seta \& Seta, 1993) in suggesting that exposure to stereotypically inconsistent behavior often invokes a motive to compensate for this deviance by expecting hyperconsistent behavior on the part of another group member, thereby increasing the weight of attributions that are consistent with the disposition of the actor. In these studies, both the aberrant actor and the fellow group members were related via their common group membership. The theoretical rationale for the obtained predictions of extreme attributions vis-à-vis a fellow group member was based on the assumption that the attributional process was biased by motives to maintain the integrity of the group concept in the face of observed inconsistency. In an attempt to 
accomplish this, perceivers generated attributions that were consistent with those of the target's group.

It follows from this perspective that such results should only be obtained when the attributional target (e.g., fellow group member) shares a perceived unit relationship with the deviant actor. That is, if patterns of attributions are colored by motives to maintain existing concepts, then these motives would only be operative when such attributions are potentially functional in meeting the maintenance goal. Please note, we are not assuming that such attributions result in the successful maintenance of extant stereotypes; they may or may not result in stereotype maintenance. Rather, the assumption in this and our prior work is that attributional and expectation-generation processes (and even behavior) are biased in the direction of stereotype/concept maintenance. If this reasoning is correct, we should observe these biases only in contexts in which attributional targets are related to the aberrant targets; for example, when they are members of the stereotyped group "under threat."

However, if some sort of perceptual contrast is operative in the setting, then the attributional target would be expected to appear more extreme in contrast to the aberrant actor regardless of his or her relationship to the actor. That is, if exposure to a deviant group member leads individuals to perceive a subsequent target in a more extreme light in contrast to the actor, any distinct target may be subject to this process (e.g., Martin \& Seta, 1983; Stapel \& Spears, 1996).

Study 3 assessed this prediction by varying whether the attributional target did or did not share a group unit relationship with an aberrantly behaving group member. The target was either a member of the actor's group or was not a fellow group member. If perceptual contrast effects underlie observed attributional patterns, then both the fellow group and different group member target conditions should show more extreme attributions than controls. However, if compensatory motivations are operative, then extreme attributions should be evidenced in the fellow group member versus the different group member condition. 


\section{METHOD: EXPERIMENT 3}

\section{Participants and Design}

Seventy-two female students from introductory psychology classes served as participants and received partial credit for their research requirements. The design factorially crossed two levels of inconsistency (no inconsistency control and an inconsistent experimental condition) with two levels of fellow group member target identity (same group, different group).

\section{Procedure}

Participants were oriented to the experiment in a manner identical to the previously described methods. Inconsistent information was given about a nurse who was "red faced, grasping a small child by the collar and slapping him painfully in the face": the inconsistent condition. This information was not presented to control condition participants. Participants read that a member of the community asked a different nurse (in the same group identity condition) or asked a salesperson (in the inconsistent different group identity condition) to donate the better part of a Saturday to help an organization while in the presence of others. All participants were asked the likelihood that this person donated time because she was truly a generous person or because she simply wanted to impress. Participants rated their attributions along a 7-point scale in which a 1 indicated that the person volunteered because she simply wanted to impress, whereas a 7 indicated that the person volunteered because she was truly generous. Control nurse and control salesperson participants were asked the same question. The only difference was that they were not exposed to the inconsistent information concerning a nurse's inconsistent behavior. 6

\section{RESULTS AND DISCUSSION: EXPERIMENT 3}

Participants' rating scores were entered into a $2 \times 2$ between-subjects analysis of variance. Table 1 contains the means of the four experimental conditions. The analysis revealed a significant Fellow Group Member Identity (same group or different group) $x$ Inconsistency interaction, $F(1,60)=5.55, p<.03$. Neither main effect reached significance. 
To interpret this interaction, and to test our specific predictions, we used the error term of the overall analysis to make specific comparisons. Planned comparisons indicated that, in the same group condition, participants in the inconsistent condition had ratings that were significantly more related to the internal characteristics of nurses than control participants, $F(1,60)=7.97, p<.01$. In the different group condition, participants' ratings in the inconsistent condition did not statistically differ from those of control group participants, $F(1,60)=0.21$, ns. These results add to those obtained in Experiments 1 and 2 in supporting our assumption that patterns of attributions result from perceivers attempt to maintain stereotypic views in the face of inconsistent information.

TABLE 1: Mean Attribution Rating Scores as a Function of Level of Inconsistency (no inconsistency control and an inconsistent experimental condition) and Two Levels of Fellow Group Member Identity (same group, different group)

\begin{tabular}{lcc}
\hline \multirow{2}{*}{ Identity } & \multicolumn{2}{c}{ Level of Inconsistency } \\
\cline { 2 - 3 } & No Inconsistency (M) & High Inconsistency (M) \\
\hline Same group & 3.72 & 5.04 \\
Different group & 4.03 & 3.83 \\
\hline
\end{tabular}

Perceivers made more extreme internal attributions of a related group member. They did not, however, change their attribution of a person who was not associated with the target group. This was expected because a person who is not associated with the target's group does not help maintain the target's stereotype; salespersons do not share the trait of "generosity" with the nurse stereotype. Thus, as expected, compensatory attributions were seen in the different nurse condition but not in the different group identity condition.

\section{EXPERIMENT 4}

Kunda and Oleson (1997) measured the strength of perceivers' stereotypes after providing them with information concerning a deviant group member. In one study, they found that the strength of a perceiver's stereotype was enhanced following information about an extremely deviant group member. 
Although Kunda and Oleson (1997) did not provide a direct test of their perspective, they offered two potential reasons for their effects. One reason involved perceptual contrast. Following exposure to an extremely deviant group member, they reasoned that perceivers contrasted the prototypical group member with the deviant, resulting in an especially strong stereotype (perceptual contrast also was discussed by J. J. Seta \& Seta, 1993). Their second reason involved surprise; because perceivers were surprised by the extremely deviant group member, they searched their knowledge structure for information that confirmed their original stereotype, and because they could easily construe information that confirmed their stereotype, their search resulted in an especially strong stereotype.

Although Study 3 suggests that a simple form of perceptual contrast does not account for our findings, it does not rule out the operation of active search processes for stereotype-confirming information. Indeed, similar to Kunda and Oleson (1997), our model also involves active search processes. However, we assume that active search processes follow from an implicit or explicit attempt to maintain a schema (mood, goal, or expectation). From our view, this search is initiated by perceivers' motivation to maintain a stereotype, not because they are simply surprised by the deviant's abnormal actions or characteristics.

One way to distinguish our view from that of Kunda and Oleson (1997) would be to demonstrate that perceivers who are motivated to maintain a stereotype engage in more compensation than those who have a lesser degree of motivation to maintain a stereotype. In contrast to the view of Kunda and Oleson, our analysis specifies specific compensatory motives assumed to underlie social judgments. To test this assumption, we can compare the responses of individuals who differ in their motivation to maintain their schema in the face of inconsistent information. Individuals who are especially motivated to maintain their schema also should be especially inclined to demonstrate the influence of compensatory motives following observed inconsistency.

An individual difference methodology, using individuals who were differentially motivated to maintain a group concept, has provided evidence in support of our interpretation (C. E. Seta et al., 1998). Following information about a deviant group member, highly motivated individuals demonstrated compensatory expectations, whereas individuals low in motivation did not. Although 
this study provided support for our motivation interpretation, it is not entirely clear whether these results were due to differences in perceivers' motivation or to differences in their knowledge structure. In Experiment 4, we attempted to control for these types of effects by altering participants' motivation within the experimental session. To vary motivation, some participants were given information that we expected would increase their motivation to maintain a stereotype, whereas others were given information that would decrease motivation. If perceivers' motivation to maintain their schema is a critical dimension, then individuals who were given information to bolster their motivation should demonstrate effects of compensatory motives in their social judgments in contrast to those given information that weakened this motivation.

In this experiment, we manipulated participants' levels of motivation by describing belief-maintenance as a process either that had been found or had not been found to lead individuals to be successful in life and relationships. For example, in the high-motivation condition, we told participants that research showed that persons who hold on to their beliefs in the face of disconfirming evidence are found to be successful in their relationships. In the low-maintenance condition, participants were told that holding on to beliefs in the face of disconfirming information was nonfunctional and led individuals to be unsuccessful in relationships. Onthe surface, these manipulations may appear to be rather heavy handed and artificial. One response to the criticism of artificiality is that this is an advantage of laboratory research, which allows us to construct contexts appropriate for theory testing that do not occur in the real world. But, regardless of this advantage, it is clear that differential motivations to maintain preexisting beliefs of the type used in this study do occur naturally. For example, many women believe that holding on to previous attitudes about their partners in the face of disconfirming evidence, such as abuse, will ultimately lead to a successful relationship, whereas other women more quickly revise their beliefs in the context of similar information. Although there are individual differences that underlie these orientations, contextual variables are also potent determinants of these motivations (e.g., interdependence theory).

We formulated our hypotheses so that they would not conform to potential demands that on the surface may seem to be apparent in this context. For example, in the high-motivation condition, participants were exposed to 
an actor who responded in a manner inconsistent with their extant stereotypes. If participants simply conform to instructions to maintain their preexisting views in the face of disconfirming evidence, then their responses should be similar to those of control condition participants. However, predictions made in the study called for participants in the high-motivation condition to be different from controls; specifically, these participants were expected to respond with relatively more extreme internal attributions (consistent with the character of the actor) concerning the stereotypically consistent behavior of a group member.

\section{METHOD: EXPERIMENT 4}

\section{Participants, Design, and Procedures}

Eighty-seven female students from introductory psychology classes served as participants. The general procedures were identical to those described in the other studies. The design factorially crossed two levels of motivation to maintain a stereotype (low or high) with two levels of inconsistency (no inconsistency control and an inconsistency experimental condition).

Participants were first given the motivation manipulation. In the high-motivation condition, they read that prominent researchers believed that individuals who were motivated to maintain their stereotypes were likely to be successful in life (e.g., in relationships). They were then given an example of a woman who "believes that men who belong to a particular group-a group that she has known for a long time-are generally positive." They were then told,

These researchers believe that this woman should maintain her attitude toward this group even if one of the members behaves in a negative way. They believe that she should try to maintain her attitude toward this group even if she finds that a particular group member has acted in a negative way.

In the low-motivation condition, participants read that prominent researchers believed that individuals who were motivated to maintain their stereotype were likely to be unsuccessful in life (e.g., in relationships). They were then given an example of a woman who "believes that men who belong to a particular group-a group that she has recently been acquainted with-are generally positive." They were then told that, 
These researchers believe that this woman should not maintain her attitude toward the group if one of the members behaves in a negative way. They believe that she should not try to maintain her attitude toward this group when she finds out that a particular group member has acted in a negative way.

In both motivation conditions, participants were told that the experimenter wanted them to think about how they felt about the information they just read and that the experimenter would ask them their opinion at the end of the session.

Participants in the inconsistent condition were given information identical to that described earlier about a female nursing student who slapped a child. They were then given the information about a different nursing student (Mary) who was asked to donate time to charity in the presence of other nursing students.

All participants were then asked the likelihood that this female nursing student donated her time because she is a truly generous person or because she simply wanted to impress, using the 7-point scale in which a 1 indicated that the nurse volunteered because she simply wanted to impress and 7 indicated that she volunteered because she was truly generous. No-inconsistency control participants were treated identically with the exception that they were not exposed to the inconsistent information. As in the prior studies, participants were asked the probe question concerning the identity of the rating target. 7 Following these procedures, participants were debriefed and thanked for their participation.

Results and Discussion: Experiment 4

Participants' rating scores were entered into a between-subjects ANOVA. The analysis revealed a significant Motivation $\times$ Inconsistency interaction, $F(1,71)=$ 4.33, $p<.05$. Table 2 contains the means for each of our four conditions. 8 To interpret this effect, and to test our specific predictions, we used the error term of the overall analysis to make specific comparisons. Planned comparisons indicated that participants in the high-motivation inconsistent condition had more extreme attributions (consistent with the internal character of nurses) than those in the low-motivation inconsistent condition, $F(1$, $71)=6.99, p<.02$, whereas the ratings of low- and high-motivation control participants did not differ, $F(1,71)=$ 
$.01, n s$. Furthermore, participants in the high-motivation inconsistent condition had more extreme internal attributions than those of control participants, $F(1,71)=$ $4.16, p<.05$, whereas the ratings of participants in the low-motivation inconsistent condition did not differ from that of control participants, $F(1,71)=1.22$, ns. In fact, they were somewhat lower than those of control participants.

The results of Experiment 4 are entirely consistent with our expectations. As expected, an effect of compensation on attributions was obtained in the high-motivation condition. And, as expected, this effect was not obtained in the low-motivation condition. These results argue for an interpretation in which perceivers' motivation to maintain stereotypic beliefs is a critical dimension for the generation of attributions.

\section{INTRODUCTION: EXPERIMENT 5}

Our view assumes that perceivers attempt to maintain a stereotype in the face of inconsistent information by engaging in compensation and that compensation typically occurs at the component level. That is, in an attempt to maintain a stereotype, perceivers alter their judgment of an individual who comprises membership of the group. In so doing, the overall evaluation of the target group may be maintained. However, as previously noted, this model does not assume that compensatory processes always lead to the successful maintenance of extant group concepts. Rather, the model posits that maintenance motives influence judgments about group members in a direction that is compensatory in nature.

In contrast, Kunda and Oleson's (1997) research was concerned with how the stereotype itself changes as a result of receiving inconsistent information about a target group member. In their studies, participants were first given information about a deviant group member. After evaluating the deviant group member, participants were asked about their stereotype-they were asked to judge members of the group, in general. Their assumption was that perceivers' judgment of a group member is equivalent to their judgment of the stereotype. Our view does not make this assumption. Rather, we assume that perceivers can attempt to compensate at the individual level while attempting to maintain their stereotype. Individuals can have a hyperconsistent view of a fellow group member while maintaining a different view of members in general (their stereotype). Consequently, judgments 
of a fellow group member can be significantly affected by the inconsistent actions of the target, whereas stereotypes may be largely unaffected by this information. Experiment 5 was designed to test this idea. We asked some participants about a fellow group member, whereas others were given a stereotype question about members of the group in general. This question was conceptually similar to the one used by Kunda and Oleson (1997).

TABLE 2: Mean Attribution Scores as a Function of Level of Inconsistency (no inconsistency control and an inconsistent experimental condition) and the Motivation to Maintain a Stereotype (low or high)

\begin{tabular}{lcc}
\hline \multirow{2}{*}{ Motivation } & \multicolumn{2}{c}{ Level of Inconsistency } \\
\cline { 2 - 3 } & No Inconsistency (M) & High Inconsistency (M) \\
\hline Low & 4.74 & 4.39 \\
High & 4.72 & 5.48 \\
\hline
\end{tabular}

\section{METHOD: EXPERIMENT 5}

\section{Participants, Design, and Procedure}

Seventy-eight female students from introductory psychology classes served as participants. The design included three group conditions: no inconsistency control (measuring judgments of nurses, in general) and two inconsistent target conditions-one that measured judgments of a fellow group member (inconsistent fellow group member condition) and one that measured judgments of nurses, in general (inconsistent stereotype condition).

Participants were oriented to the study in a manner identical to the previously described procedures. In this study, all participants read that there are approximately 65,000 female student nurses in the country. In the no inconsistency control condition, participants were asked to consider that "if all of the student nurses were asked to donate the better part of a Saturday to help an organization, what would be the likelihood that they would help because they are, in general, truly generous?" They responded on a 7-point scale where 1 represented not at all generous and 7 represented truly generous. Participants in the inconsistent stereotype target condition were also 
presented this information. However, before being asked the likelihood question concerning the entire population of student nurses, participants in this condition first read information about a female student nurse who slapped the child.

In the inconsistent fellow group target condition, participants were exposed to the procedures described above; however, they were not asked about their opinion of the entire population of student nurses. Rather, they were asked the likelihood that a different female nursing student would help because she is truly generous on the 7-point scale described above. In this study, our probe question simply asked whether our noncontrol participants evaluated an entire population (65,000 student nurses) or a different individual. All participants gave the correct answer.

\section{Results and Discussion:}

\section{Experiment 5}

Participants' scores were entered into a three-factor between-subjects ANOVA. The analysis revealed a significant condition main effect, $F(2,75)=5.60, p<.005$. To interpret this effect, and to test our specific predictions, we used the error term of the overall analysis to make specific comparisons. Planned comparisons indicated that participants in the inconsistent fellow group member target condition $(M=5.01)$ had more extreme attributions that were consistent with the internal character of nurses than those in the no inconsistency control $(M=$ 4.09), $F(1,75)=10.2, p<.01$. The results conceptually replicate those obtained in Experiments 1 through 4. In addition, the judgments of participants in the fellow group member target condition were significantly more consistent with the internal character of nurses than those in the inconsistent stereotype target condition $(M=$ 4.25), $F(1,75)=6.56, p<.05$. Furthermore, judgments in the inconsistent stereotype condition did not differ from those in the no inconsistency control $(M=4.09), F(1$, $75)=.31, p>.60$.

These results are entirely consistent with our predictions. Perceivers had more extreme attributions that were consistent with the character of nurses following exposure to the inconsistent actions of a deviant nurse. They did not, however, change their view of nurses, in general. Their judgments in the inconsistent stereotype condition did not differ from those in the control condition. Thus, these results suggest that judgments about a 
particular group member can reflect compensatory processes, whereas judgments about the stereotyped group per se remain relatively equivalent to conditions in which no inconsistency was encountered. Therefore, it appears that the overall evaluation of the group stereotype was maintained in the face of inconsistency. This pattern of results is quite different from that of Kunda and Oleson (1997), who found hyperconsistent judgments of the group stereotype in their research. It may be the case that Kunda and Oleson's results were obtained because participants in their study rated the person who performed the inconsistent action immediately before being asked about the group stereotype.

This procedure may have produced response scale shifts such that participants' group ratings were inflated following their previous negative evaluations of the inconsistent actor.

The present results also provide evidence that perceivers did not compare and contrast the valence of the target's actions to that of other group members. If this was the case, the judgments of participants in the inconsistent stereotype target condition should have been more extreme than that of those in the control condition.

\section{INTRODUCTION: EXPERIMENT 6}

Experiment 6 was designed to provide a test of our model's capability component. In Experiment 4 and in prior research (C. E. Seta et al., 1998), we have shown that perceivers compensate if they are motivated to maintain their stereotype. However, this does not mean that they will always be capable of accessing or generating information that can potentially compensate for all types of inconsistent actions. For example, perceivers with a certain level of motivation may be capable of compensating for the inconsistent action of one target but not those of multiple actors. Experiment 6 was designed to determine if perceivers are capable of compensating for the inconsistent actions of one target nursing student, but not those of multiple nursing students. In addition to supporting our capability assumption, the expected results also would provide additional evidence against an interpretation in which perceivers compare the valence of the target's behavior to that of a fellow group member. If this type of contrast is occurring, perceivers should see the action of a fellow group member as especially positive after observing the inconsistent actions of both multiple and individual targets. 


\title{
METHOD: EXPERIMENT 6
}

\author{
Participants, Design, and Procedures
}

Seventy-seven female students from introductory psychology classes served as participants and received partial credit for their research requirements.9 The design included three group conditions: a no inconsistency control and two inconsistent group conditions. In the two inconsistent group conditions, participants read either about the inconsistent actions of a single group member (single actor condition) or about multiple actors (multiple actor condition). The orientation of participants in this study was identical to that of the previous studies. However, participants in the multiactor inconsistent condition read about a nursing student who slapped a child and a different nursing student who taunted an old man. Participants in the single actor inconsistent condition were presented these inconsistent actions as performed by one nursing student. Participants in both inconsistency conditions were asked about the likelihood of a different nurse donating her time, in the manner described in the previous studies. Control participants were asked the same question but were not exposed to inconsistent information.

\section{RESULTS: EXPERIMENT 6}

Participants' rating scores were entered into a three-factor between-subjects analysis of variance. The analysis revealed a significant group main effect, $F(2,56)=6.01$, $p<.01$. To interpret this effect, and to test our specific predictions, we used the error term of the overall analysis to make specific comparisons. Planned comparisons indicated that participants in the inconsistent single actor condition ( $M=5.53)$ had more extreme attributions that were consistent with the disposition of nurses than those in the control condition $(M=4.24), F(1,56)=$ $9.32, p<.01$, whereas the attributions of participants in the multiple actor condition $(M=4.26)$ did not differ from that of control participants, $F(1,56)=.0001$, ns.

\section{DISCUSSION: EXPERIMENT 6}

The results of Experiment 6 are entirely consistent with our expectations. First, we found that participants in the single group member actor condition had more extreme attributions that were consistent with the internal character of nurses than those in the control condition. 
The novel finding in this study involved the ratings of participants in the multiple group member actor's condition. The attributions of these participants did not differ from those of controls, and they were significantly less consistent with the internal character of the actors than those of participants in the singular group member actor condition. Thus, when participants were exposed to the inconsistent actions of two different nurses, they did not interpret a fellow group member's behavior in a compensatory manner. Therefore, just as participants in Experiments 1 and 2 did not interpret the target's behavior in a compensatory manner following the target's own inconsistent actions, participants in Experiment 6 did not interpret the actions of a fellow group member in a compensatory manner after observing the inconsistent actions of two different targets.

These data suggest that it may take a larger number of inconsistent actions to constrain compensation in the context of a fellow group member (e.g., it took the inconsistent actions of two target persons) than to constrain compensation in the context of the person performing the inconsistent actions (e.g., it only took the inconsistent actions of the target to constrain compensation in Experiments 1 and 2). As discussed in the introduction, there are several reasons why a perceiver can expect compensation from a fellow group member but not from the target of the inconsistent action. First, the assumed variability among members is often greater than the assumed variability in a single individual's behavior (e.g., Hamilton \& Sherman, 1996). In addition, the implications of inconsistent actions are more directly tied to the personality of the target of the inconsistent action than to the personality of a fellow group member. Thus, it follows that it should take an objectively less severe inconsistent action (or fewer inconsistent actions) to constrain a perceiver's capability of generating compensatory expectations and attributions about the acting target versus a fellow group member. In addition, these results provide evidence that participants are not comparing the valence of the target to a prototype member of the group. If this interpretation accounted for our effects, participants should have viewed the behavior of a fellow group member as especially consistent with the character of nurses after observing the highly inconsistent actions of multiple targets.

This was not the case. 


\section{GENERAL DISCUSSION}

Attributions play an important role in theoretical accounts of social behavior, and attributional processes have been the subject of considerable research (see Fiske \& Taylor, 1991, for a review). The results of the present research add to this body of knowledge by demonstrating an influence of compensatory motivation on patterns of attribution. In particular, results show that perceivers are biased toward relatively extreme stereotypical internal attributions of group members' behaviors when previously exposed to behaviors that are inconsistent with stereotypic expectations. These attributions could potentially compensate for the inconsistency. Thus, attributional processes were shown to be biased by motives to maintain stereotype concepts. The present research also complements existing research on attributional mechanisms of stereotype maintenance. For example, the type of attribution a perceiver makes about an inconsistent action can influence whether a person who behaves in an aberrant manner is subtyped, that is, seen as an "exception to the rule" (e.g., Crocker et al., 1983; Johnston \& Hewstone, 1992; Weber \& Crocker, 1983). Subtyping and compensation can work in tandem. It should be pointed out, however, that whereas subtyping and compensation can work in tandem, compensation does not require subtyping —as has been shown, for example, by the finding that perceivers can compensate via the target himself or herself (J. J. Seta \& Seta, 1993).

The results of the present studies demonstrate that interpretations of the behavior of group members who are related to targets only by virtue of category membership also are influenced by the inconsistent behavior of the deviant. Thus, this research demonstrates that the aberrant behavior of a deviant group member not only affects attributions about that person's behavior but also affects attributions about other members of the stereotyped group. This finding has important implications for the resistance of stereotypes to change, which will be elaborated on further in this article. In brief, because the assumed variability among members of a group is often greater than the assumed variability in a single individual's behavior, the generation of compensation at the group level is possible when it may not be possible at the individual level. Furthermore, the implications of inconsistent actions are tied more directly to the personality of the target of the inconsistent actions than to the personality of fellow group members. Thus, these processes may constitute a strong force against stereotype change. 
Study 5 demonstrated that judgments about individual group members may not be identical to stereotypic judgments about members of the group in general. In this study, we used a stereotype measure that was conceptually similar to the one used by Kunda and Oleson (1997). From our model, individuals may exhibit extreme compensatory judgments about members of a group while maintaining their extant group stereotype. That is, attributions and expectations concerning a particular group member may reflect compensatory processes, whereas judgments about the stereotyped group per se remain equivalent to conditions in which no inconsistency is observed. In addition to ruling out simple contrast explanations for these findings, this result suggests that compensatory processes do potentially serve the function of maintaining, while not necessarily strengthening, existing stereotypes.

The results of Experiment 6 demonstrated that inconsistent actions on the part of several group members constrain perceivers' capability of compensating via different members of stereotyped groups. In Experiment 6 , the ratings of participants in the multiple actor inconsistent condition were not more compensatory than control group participants, and they were less compensatory than those of participants in the inconsistent single actor condition.

This research also demonstrated that the generation of compensatory attributions involves motivation. For example, in Study 4, priming or framing participants with arguments pro or con the utility of maintaining/ changing extant beliefs had the effect of influencing the generation of compensatory attributions.

\section{Contrast and Compensation}

These experiments rule out various alternative interpretations. They, for example, rule out a contrast interpretation in which perceivers compare the target to a prototypic member of the group. It should be pointed out, however, that contrast (and assimilation) can be used as descriptive terms. Contrast refers to the fact that one stimulus moves away from another, whereas assimilation refers to the fact that one stimulus moves toward another. If contrast is used in a descriptive manner, then a person may want to substitute the term motivated contrast for compensation in our analysis while maintaining our key assumptions. For example, perceivers, to maintain their stereotype, can be viewed as contrasting the fellow 
group member to the target. In this case, people are motivated to move their evaluations and valuations of a fellow group member away from the group mean to maintain the stereotype. This will occur if perceivers are motivated to maintain their stereotype and if they can generate or access information that is compensatory in nature. In this case, motivated contrast is being used in place of compensation. It is being used to portray a process by which perceivers compensate for one inconsistency by altering another action. If contrast is used in this way (i.e., by incorporating our key assumptions), then it is not necessary to tease apart a contrast interpretation (or other interpretations with similar meanings) from our schema maintenance through compensation analysis. We have chosen the word compensation (e.g., J. J. Seta \& Seta, 1992, 1993) instead of motivated contrast because compensation implies that a person is motivated to counterbalance an observed inconsistency.

Implications for the resistance of stereotypes and attitudes. Not only do the results of our experiments support the idea that perceivers compensated in an attempt to maintain their stereotype, but the results of Experiment 5, in particular, suggest that compensation may result in successful stereotype maintenance. Following an inconsistent action on the part of a group member, perceivers evaluated a different behavior of a different group member in a hyperconsistent manner. However, using a stereotype measure that was conceptually similar to one used by Kunda and Oleson (1997), we found that a separate group of participants did not change their stereotype following observed inconsistency. Assuming that these participants also would have generated compensatory attributions if assigned to the different group member target condition (e.g., that there were no failures of random assignment), this result supports the idea that compensation can result in successful stereotype maintenance. It also implies that compensation can help prevent different types of schema from changing, such as goals and attitudes.

After exposure to the inconsistent actions of a target group member, perceivers can maintain their overall group stereotype in a number of different ways. One way is to modify their expectations and attributions about how and why a different group member performed the same behavior as the target; to balance the inconsistent actions of a target, the different group member's actions are perceived to be hyperconsistent. In this way, perceivers are maintaining the content as well as the overall evaluative nature of their stereotype. The overall 
evaluative nature of a nurse stereotype, for example, is maintained if one component of the stereotype balances a different component. Although the overall evaluative nature of nurses may be maintained, exposure to inconsistency and the generation of compensatory judgments also might lead to changes in stereotype content or variability.

\section{Summary}

The results of all six experiments provide support for the key assumptions of our schema-maintenance through compensation analysis. In addition, they add to the body of knowledge concerning attributions in demonstrating the role of compensatory motivation on patterns of attributions.

\section{Caveats}

In this article, we have discussed situations in which individuals were attempting to keep their view of a particular group from changing. However, a schema can be dynamic, such as when a person is interested in becoming progressively better and better at a particular skill (e.g., Wood, 1989). In this situation, because the goal is improvement, we are attempting to maintain a situation in which our outcomes and activities are progressively more and more positive. So, for example, if we are attempting to maintain the goal of increasing our net worth each year, we will attempt to generate activities that will satisfy this upwardly mobile goal.

Although research has not explicitly dealt with a schema of the sort we have just discussed, it has dealt with situations in which the schema was related to the activities of the perceiver rather than to the activities of other individuals (e.g., J. J. Seta et al., 1993; J. J. Seta \& Seta, 1982). Therefore, our analysis is applicable to situations in which the perceiver is the actor as well as the observer. 


\section{NOTES}

1. There are circumstances in which schema change is desirable.

For example, at times we have a goal to self-improve (e.g.,Wood, 1989). In these situations, because the goal is improvement, we are attempting to maintain change rather than the status quo.We will discuss situations like this in the General Discussion.

2. It should be pointed out that our model makes relative and not absolute predictions. For example, we cannot predict the exact level of an inconsistent event (or the exact number of inconsistent events) that will constrain perceivers' capability of compensating.We can, however, make relative predictions about the conditions that make it more or less likely to compensate.

3. The data of 7 participants were not included because they did not properly identify the person they were evaluating. The materials presented in this and subsequent studies were complex, and if participants did not pay careful attention they misread or confused the information.

4. Six participants indicated that they majored in nursing and were excluded from the study. Also, 6 people were excluded from our study for giving the incorrect answer on our probe question.

5. Perceivers may have believed that the actions of the inconsistently behaving nurse who was asked to volunteer were known to the other fellow group members. If the actions of the inconsistently behaving nurse were known, the fellow group member might be especially inclined to want to impress (e.g., have self-presentational motives). To rule out this possibility, we gave the scenarios used in this research to a separate group of participants. These participants $(n=18)$ were asked whether the fellow group member nurse would want to impress a group of other nurses-after they witnessed the inconsistent target's behavior-in contrast to a situation in which the information about the inconsistently behaving nurse was not known. Ona 5-point scale where 1 represented wants to impress most when information was known, 5 represented wants to impress most when information was not known, and 3 represented no difference, results revealed no difference in estimates of "volunteering to impress" across these two situations $(M=3.2$, where 3 represented no difference), $F(1,17)=.25$, ns. Thus, explanations based on the idea that the manipulation either increased or decreased assumed self-presentational motives are not viable.

6. Eight participants were excluded from the study for giving the incorrect answer on our probe question, which was conceptually similar to the one used in Experiment 1.

7. Twelve people were excluded from our study for giving the incorrect answer on our probe question, which was conceptually similar to the one used in Experiment 2. 
8. Methodological changes incorporated into Study 4, including group salience and name changes, most probably accounted for discrepancies between the control groups in this study and the controls in our previous nursing studies.

9. Four participants indicated that they majored in nursing and were excluded from the study. Also, 14 participants gave an incongruent answer for the question asking them about the person that they evaluated and were excluded from the study.

\section{REFERENCES}

Allport, G. W. (1954). The nature of prejudice. Reading, MA: Addison Wesley.

Crocker, J., Hannah, D. B., \& Weber, R. (1983). Person memory and causal attributions. Journal of Personality and Social Psychology, 44, 55-66.

Fiske, S. T., \& Neuberg, S. L. (1990). A continuum of impression formation, from category-based to individuating process: Influences of information and motivation on attention and interpretation. In M. P. Zanna (Ed.), Advances in experimental social psychology (Vol. 23, pp. 1-74). New York: Academic Press.

Fiske, S. T., \& Taylor, S. E. (1991). Social cognition (2nd ed.). New York: McGraw-Hill.

Hamilton, D. L., \& Sherman, S. J. (1996). Perceiving persons and groups. Psychological Review, 103, 336-355.

Heider, F. (1958). The psychology of interpersonal relations. New York: John Wiley.

Hewstone, M. (1989). Causal attribution. Cambridge, MA: Basil Blackwell.

Johnston, L., Bristow, M., \& Love, N. (2000). An investigation of the link between attributional judgments and stereotype-based judgments. European Journal of Social Psychology, 30, 551-568.

Johnston, L., \& Hewstone, M. (1992). Cognitive models of stereotype change. Subtyping and the perceived typicality of disconfirming group members. Journal of Experimental Social Psychology, 28, 360386. 
Jones, E. E., \& Davis, K. E. (1965). From acts to dispositions: The attribution process in person perception. In L. Berkowitz (Ed.), Advances in experimental social psychology (Vol. 2, pp. 220-266). New York: Academic Press.

Kelley, H. H. (1973). The processes of causal attribution. American Psychologist, 28, 107-128.

Kunda, Z., \& Oleson, K. C. (1997). When exceptions prove the rule. How extremity of deviance determines the impact of deviant examples on stereotypes. Journal of Personality and Social Psychology, 72, 965-979.

Martin, L. L., \& Seta, J. J. (1983). Perceptions of unity and distinctiveness as determinants of attraction. Journal of Personality and Social Psychology, 44, 755-764.

Moreno, K., \& Bodenhausen, G. (1999). Resisting stereotype change: The role of motivation and attentional capacity in defending social beliefs. Group Processes and Intergroup Relations, 2, 5-16.

Seta, J. J.,\&Seta, C. E. (1982). Personal equity: An intrapersonal comparator system analysis of reward value. Journal of Personality and Social Psychology, 43, 222-235.

Seta, J. J.,\&Seta, C. E. (1992). Personal equity-comparison theory: An analysis of value and the generation of compensatory and noncompensatory expectancies. Basic and Applied Social Psychology, 13, 47-66.

Seta, J. J.,\&Seta, C. E. (1993). Stereotypes and the generation of compensatory and noncompensatory expectancies of group members. Personality and Social Psychology Bulletin, 19, 722-731.

Seta, J. J., Seta, C. E., \& Erber, M.W. (1993). The role of cost in generating expectations and value: A personal equity comparison theory analysis. Basic and Applied Social Psychology, 14, 103-119.

Seta, C. E., Seta, J. J.,\&Goodman, R.C. (1998). Social identity orientation and the generation of compensatory expectancies. Basic and Applied Social Psychology, 20, 280-295.

Stapel, D. A., \& Spears, R. (1996). Event accessibility and context effects in causal inference: Judgment of a different order. Personality and Social Psychology Bulletin, 22(10), 979-992.

Weber, R., \& Crocker, J. (1983). Cognitive processes in the revision of stereotypic beliefs. Journal of Personality and Social Psychology, 45, 961-977. 
Wilder, D. A., Simon, A. F., \& Faith, M. (1996). Enhancing the impact of counterstereotype information: Dispositional attributions for deviance. Journal of Personality and Social Psychology, 71, 276-287.

Wilson,T. D., Lisle, D. J., Kraft, D.,\&Wetzel, C. G. (1989). Preferences as expectation-driven inferences: Effects of affective expectations on affective experiences. Journal of Personality and Social Psychology, $56,519-530$.

Wood, J. V. (1989). Theory and research concerning social comparisons of personal attributes. Psychological Bulletin, 106, 231-248.

Yzerbyt, V. Y., Coull, A., \& Rocher, S. (1999). The role of cognitive resources in the maintenance of stereotypes. Journal of Personality and Social Psychology, 77, 449-462.

Yzerbyt, V. Y., Rogier, A., \& Fiske, S. (1998). Groups entitativity and social attributions: On translating situational constraints into stereotypes. Personality and Social Psychology Bulletin, 24, 1089-1103. 